\title{
离子液体中卤代炔烃高效水解制取 $\alpha$-卤代甲基酮的研究
}

\author{
付文强 ${ }^{a}$ 谭 平 $b$ 邓伟 $a$ 向建南*,a

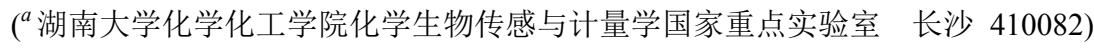 \\ ( ${ }^{b}$ 湖南机电职业技术学院 长沙 410151)
}

\begin{abstract}
摘要 在离子液体中, 硫酸促进卤代炔烃水解生成 $\alpha$-卤代甲基酮，反应收率高达 $85 \% \sim 94 \%$. 离子液体-硫酸反应体系 可多次 (5 次以上)循环使用而不影响反应收率. 同时，此反应体系底物适用性强，高效制得一系列芳环上不同取代基的 (如甲基、甲氧基、羟基、硝基等) $\alpha$-氯/溴/碘代苯乙酮及脂肪类卤代甲基酮.
\end{abstract}

关键词＼cjkstart离子液体; 卤代炔烃; 水解; $\alpha$-卤代甲基酮; 可循环

\section{Efficient Hydrolysis of Haloalkynes to $\alpha$-Haloketones in Ionic Liquid}

\author{
$\mathrm{Fu}$, Wenqiang ${ }^{a} \quad{\text { Tan, } \text { Ping }^{b} \quad \text { Deng, }^{b} \mathrm{We}^{a} \quad \text { Xiang, Jiannan }}^{*, a}$ \\ $\left({ }^{a}\right.$ State Key Laboratory of Chemo/Biosensing and Chemometrics, College of Chemistry and Chemical Engineering, \\ Hunan University, Changsha 410082) \\ $\left({ }^{b}\right.$ Hunan Mechanical and Electrical Polytechnic, Changsha 410151)
}

\begin{abstract}
The haloalkynes were hydrolyzed to $\alpha$-haloketones by sulfuric acid promotion in ionic liquids (ILs) with $85 \%$ $94 \%$ yields. The $\mathrm{ILs}_{-} \mathrm{H}_{2} \mathrm{SO}_{4}$ reaction system could be easily recycled (more five times) without any effect for reaction yield. At the same time, a wide scope of substrates haloalkynes were proper in this reaction system and a series of $\alpha$-chloro/bromo/iodo acetophenones with different substituent (such as methyl, methoxyl, hydroxyl, nitro etc.) and aliphatic $\alpha$-haloketones have been obtained in good yields.
\end{abstract}

Keywords ionic liquid; haloalkynes; hydration; $\alpha$-haloketones; recyclable

由于 $\alpha$-卤代甲基酮在构建具有生物活性的杂环化 合物 ${ }^{[1]}$ 和天然产物 ${ }^{[2]}$ 方面具有广泛的应用, 而引起人们 关注. 除此之外, 一些 $\alpha$-卤代甲基酮本身就具有生物活 性, 常常应用于药物或者辅助药物, 例如酶抑制剂 ${ }^{[3]}$. 传统的关于制得 $\alpha$-卤代甲基酮报道的方法有很多, 大多 是利用卤素或者相关卤代试剂与烯烃、酮等发生卤化反 应 ${ }^{[4]}$. 然而, 这些反应都具有一定的局限性. 例如, 使用 到有毒和腐蚀性极强的卤素单质、反应时间长、条件苛 刻、易形成过卤化物等. 为了克服这些问题, 本课题组 开创了利用 $\mathrm{Au}^{[5]} 、 \mathrm{Cu}^{[6]}$ 等金属催化剂催化卤代炔烃水解 制得 $\alpha$-卤代甲基酮的新方法(图 1). 随之使用金属催化 剂如 $\mathrm{Ag}^{[7 \mathrm{a}]} 、 \mathrm{II}^{[7 \mathrm{~b}]}$ 催化卤代炔烃水解的方法得到发展. 虽 然上述反应有各自的优点, 但是金属催化剂价格昂贵, 不便于回收, 对环境造成污染等弊端显得十分突出. 因
此发展一种新的成本低廉、绿色无污染制取 $\alpha$-卤代甲基 酮的方法很有必要.

离子液体反应体系在有机合成方面具有许多的优 良性能而被广泛关注 ${ }^{[8]} .2011$ 年, Wong 等 ${ }^{[9]}$ 报道了一种 新型离子液体反应体系对水解反应具有很好的催化促 进作用. 因此, 作者合成了该新型离子液体, 研究了卤 代炔烃在该新型离子液体水解反应及反应体系的循环 使用情况，创建了一种在离子液体反应体系中卤代炔烃 水解生成 $\alpha$-卤代甲基酮的新方法. 该方法具有反应效率 高、时间短、条件温和、不需要加入金属催化剂等优点, 同时, 该催化反应体系可循环使用, 绿色经济无污染.

\section{1 结果与讨论}

参考 Wong 的方法, 合成了这种离子液体 $\mathbf{1} \cdot\left(\mathrm{HSO}_{4}\right)_{2}$

* Corresponding author. E-mail: jnxiang@hnu.edu.cn

Received October 18, 2016; revised December 30, 2016; published online January 20, 2017.

Project supported by the Planned Science and Technology Project of Hunan Province (No. 2015WK3003), the Scientific Research Fund of Hunan Provincial Education Department (No. 14C0405).

湖南省科技厅基金(No. 2015WK3003)、湖南省教育厅科研基金(No. 14C0405)资助项目. 
(Eq. 1).

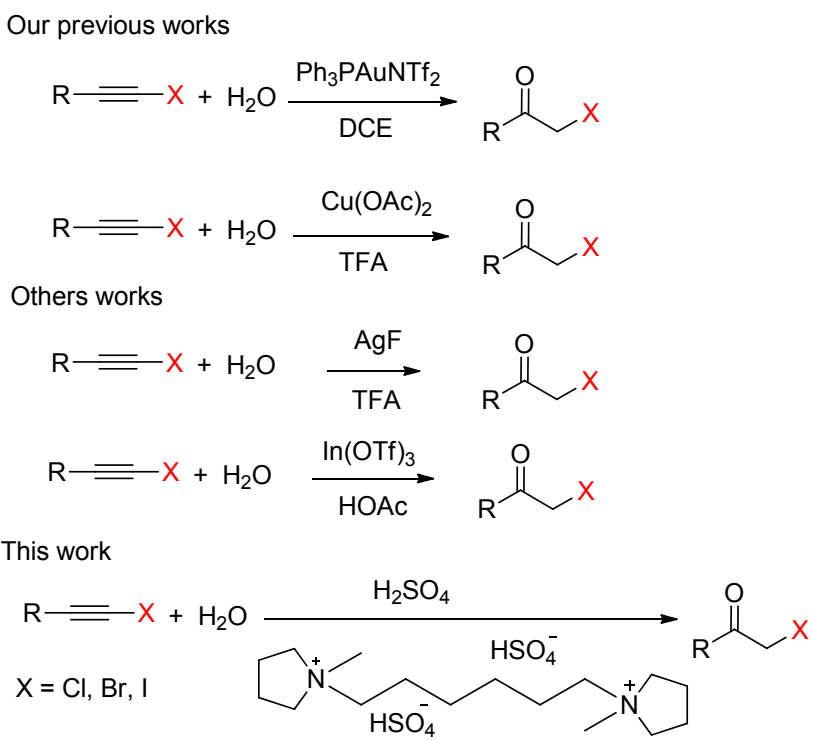

图 1 卤代炔烃水解的几种方法

Scheme 1 Methods for hydration of haloalkynes

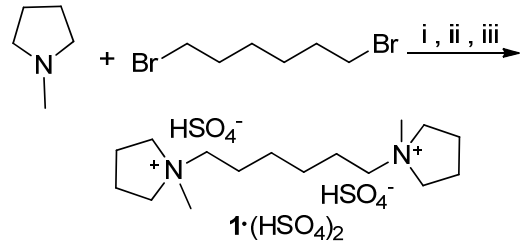

Reagents and conditions: (i) Reflux in acetonitrile to give $\mathbf{1} \bullet(\mathrm{Br})_{2}$; (ii) removal of the bromide anions by $\mathrm{Ag}_{2} \mathrm{O}$ in water at room temperature to give $1 \cdot(\mathrm{OH})_{2}$; (iii) neutralization of $1 \cdot(\mathrm{OH})_{2}$ with $\mathrm{H}_{2} \mathrm{SO}_{4}$ in water to give $1 \cdot\left(\mathrm{HSO}_{4}\right)_{2}$

用离子液体 $\mathbf{1} \cdot\left(\mathrm{HSO}_{4}\right)_{2}$ 作为反应介质, 以苯乙炔溴 (1a)为模型底物对反应的条件进行了优化, 通过对温 度、硫酸加入量、反应物配比等条件的篮选, 确定了反 应的最优条件, 实验结果见表 1.

从表 1 可知, 最初 $0.5 \mathrm{mmol}$ 苯乙炔溴、 $0.5 \mathrm{mmol}$ $\mathrm{H}_{2} \mathrm{O}$ 加入到 $3.5 \mathrm{mmol}$ 的离子液体 $\mathbf{1} \cdot\left(\mathrm{HSO}_{4}\right)_{2}$ 中, 加入 7.5 $\mathrm{mmol} \mathrm{H}_{2} \mathrm{SO}_{4}$ 在室温下反应 $2 \mathrm{~h}$ 得到 $\alpha$-溴代甲基酮(2a)的 收率为 $23 \%$. 首先探索了温度对反应收率的影响, 当反 应体系的温度从室温升高至 $40 、 70{ }^{\circ} \mathrm{C}$ (表 1 , Entries 2,3) 反应收率在 $70{ }^{\circ} \mathrm{C}$ 时最高达到 $68 \%$; 温度继续升高至 $90{ }^{\circ} \mathrm{C}$ (表 1, Entry 4), 产率反而降低, 这是因为温度过 高, 反应物水会蒸发冷凝在反应瓶口附近, 影响反应收 率. 随后考察了硫酸量对反应收率的影响(表 1, Entries $5 \sim 8)$, 当没有硫酸加入时, 该反应不能发生; 随着加入 $\mathrm{H}_{2} \mathrm{SO}_{4}$ 量的增加, 反应收率增加, 发现当加至 8 equiv. (表 1, Entry 8), 反应收率达 69\%不再随之上升. 最后对 反应底物苯乙炔澳(1a)与 $\mathrm{H}_{2} \mathrm{O}$ 的配比研究发现: 当 1a 与 $\mathrm{H}_{2} \mathrm{O}$ 为 $1: 2$ (物质的量比)时, 反应收率高达 $93 \%$.
表 1 反应条件筛选 ${ }^{a}$

Table 1 Screening of the reaction conditions

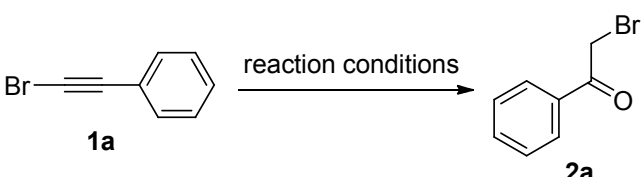

$2 a$

\begin{tabular}{cccccc}
\hline Entry & Water $/ \mathrm{mmol}$ & $\mathrm{Temp} .{ }^{\circ} \mathrm{C}$ & $\mathrm{Time} / \mathrm{h}$ & $\mathrm{H}_{2} \mathrm{SO}_{4} / \mathrm{mmol}$ & $\mathrm{Yield}^{b} / \%$ \\
\hline 1 & 0.5 & r.t. & 2 & 7.5 & 23 \\
2 & 0.5 & 40 & 2 & 7.5 & 56 \\
3 & 0.5 & 70 & 2 & 7.5 & 68 \\
4 & 0.5 & 90 & 2 & 7.5 & 46 \\
5 & 0.5 & 70 & 2 & 0 & 0 \\
6 & 0.5 & 70 & 2 & 1 & 32 \\
7 & 0.5 & 70 & 2 & 2 & 52 \\
8 & 0.5 & 70 & 2 & 4 & 69 \\
$\mathbf{9}$ & $\mathbf{1}$ & $\mathbf{7 0}$ & $\mathbf{2}$ & $\mathbf{4}$ & $\mathbf{9 3}$ \\
10 & 2 & 70 & 2 & 4 & 90 \\
11 & 1 & 70 & 1 & 4 & 75 \\
12 & 1 & 70 & 4 & 4 & 93 \\
\hline
\end{tabular}

${ }^{a}$ Reaction conditions: 1a $(0.5 \mathrm{mmol})$ in $\mathbf{1} \cdot\left(\mathrm{HSO}_{4}\right)_{2}(3.5 \mathrm{mmol}) ;{ }^{b}$ Estimated by ${ }^{1} \mathrm{H}$ NMR spectroscopy using diethyl phthalate as an internal reference.

TLC 监测反应时间，发现 $2 \mathrm{~h}$ ，可完成反应.通过上述对 反应体系的优化，最佳反应条件是在离子液体中 $70{ }^{\circ} \mathrm{C}$, 反应物及硫酸配比为 $1 \mathrm{a}: \mathrm{H}_{2} \mathrm{O}: \mathrm{H}_{2} \mathrm{SO}_{4}=1: 2: 8$ (物质 的量比).

在最佳反应条件下，探索了这种反应体系的可循环 性, $10 \mathrm{mmol}$ 苯乙炔溴、 $20 \mathrm{mmol} \mathrm{H}_{2} \mathrm{O}$ 加入到 $70 \mathrm{mmol}$ 的离子液体 $\mathbf{1} \cdot\left(\mathrm{HSO}_{4}\right)_{2}$ 中, 加入 $80 \mathrm{mmol} \mathrm{H}_{2} \mathrm{SO}_{4}$ 进行反 应. 反应结束冷却至室温, 反应产物用乙酸乙酯萃取用 于反应收率计算，离子液体- $\mathrm{H}_{2} \mathrm{SO}_{4}$ 体系真空干燥 $24 \mathrm{~h}$ 后直接用于下一次循环. 在下一次循环中, 只需加入 10 $\mathrm{mmol}$ 苯乙炔溴、 $20 \mathrm{mmol} \mathrm{H}_{2} \mathrm{O}$ 在相同条件下进行反应. 通过 5 次实验，每次循环中反应产率都在 $90 \%$ 以上. 因 此，这种反应体系十分稳定，可在催化卤代炔烃水解反 应中循环使用。

在最优的反应条件下, 选用不同的卤代炔烃进行了 底物适用性的研究, 实验结果如表 3. 实验结果表明, 该反应体系具有广泛的底物适用性. 无论在苯环上的取 代基是供电子基团(如 $2 \mathbf{c} ， 89 \%$ )还是吸电子基团(如 $2 \mathbf{f}$, $88 \%$ ), 无论在对位(如 $\mathbf{2 b}, 93 \%$ )、间位(如 $\mathbf{2 m}, 91 \%$ )、邻 位(如 2o, 90\%)，反应都具有极高的收率，也说明该反应 中苯环上取代基的电子效应和空间效应对该反应的影 响不大．同时，脂肪类及杂环类卤代炔烃，同样适用于 该反应体系(如 $2 \mathrm{u}, 2 \mathrm{v}, \mathbf{2 w}$ ). 另外，我们发现不仅苯乙炔 溴可以发生该反应，苯乙炔氯(2s，94\%)、苯乙炔碘( $2 \mathrm{t}$, $85 \%$ )同样可以以较高的反应收率获得相应的产物，且 卤素原子吸电子能力越强, 相应产物产率越高. 
表 3 反应适用性研究 $a, b$

Table 3 Reaction scope

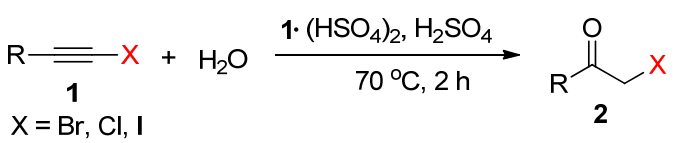

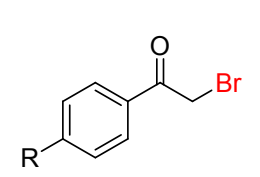

2a, $\mathrm{R}=\mathrm{H}, 91 \%$

2b, $R=M e, 93 \%$

2c, $\mathrm{R}=\mathrm{MeO}, 89 \%$

2d, $\mathrm{R}=\mathrm{Ph}, 90 \%$

2e, $\mathrm{R}=\mathrm{AcO}, 87 \%$

2f, $R=F, 88 \%$

2g, $\mathrm{R}=\mathrm{Cl}, 89 \%$

2h, $R=1,88 \%$

2i, $\mathrm{R}=\mathrm{CF}_{3}, 86 \%$

2j, $\mathrm{R}=\mathrm{NO}_{2}, 87 \%$

2k, $\mathrm{R}=\mathrm{CN}, 86 \%$

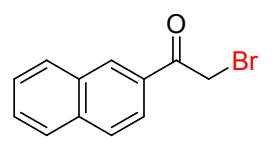

2q, $90 \%$<smiles>O=C(CI)c1ccccc1</smiles>

$2 t, 85 \%$

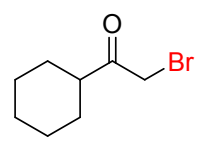

2v, $87 \%$

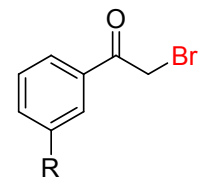

2l, $\mathrm{R}=\mathrm{Br}, 88 \%$

2m, R = Me, $91 \%$

2n, $\mathrm{R}=\mathrm{OH}, 89 \%$

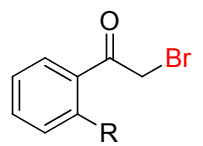

2o, $\mathrm{R}=\mathrm{Me}, 90 \%$

2p, $\mathrm{R}=\mathrm{Cl}, 91 \%$

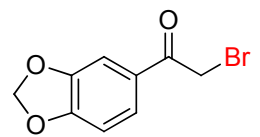

2 r, $88 \%$

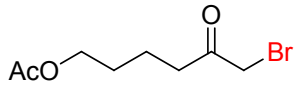

$2 u, 89 \%$

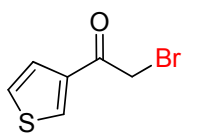

2 w, $85 \%$
${ }^{a}$ Reaction conditions: $\mathbf{1}(0.5 \mathrm{mmol}), \mathrm{H}_{2} \mathrm{O}(1.0 \mathrm{mmol}), \mathbf{1} \cdot \mathrm{HSO}_{4}(3.5 \mathrm{mmol})$, $\mathrm{H}_{2} \mathrm{SO}_{4}(4.0 \mathrm{mmol}){ }^{b}$ Isolated yields.

\section{2 结论}

本文发展了一种新的 $\alpha$-卤代甲基酮的制备方法. 在 离子液体-硫酸反应体系中卤代炔烃与水以 $1: 2$ 的物质 的量比，85\% 94\%的收率生成 $\alpha$-卤代甲基酮. 该方法 不需要使用金属催化剂, 原料便宜易得, 反应条件温和 $\left(70{ }^{\circ} \mathrm{C}\right)$, 反应效率高、时间短 $(2 \mathrm{~h})$. 此外, 该反应催化 体系可循环使用，具有经济环保等优点.

\section{3 实验部分}

\section{1 仪器与试剂}

所有原料均购于百灵威科技有限公司、萨恩一安耐 吉化学技术(上海)有限公司等, 未进一步处理. ${ }^{1} \mathrm{H}$ NMR (400 MHz) 和 ${ }^{13} \mathrm{C}$ NMR (100 MHz) 使用 Brucker ARX 400
FT NMR $\left(\mathrm{CDCl}_{3}\right.$ 为溶剂, TMS 为内标物)型核磁共振仪 测定. SGW-1 X-4 显微熔点仪测定熔点. 柱层析用硅胶 $200 \sim 300$ 目.

\section{2 实验方法}

卤代炔烃水解的一般方法

在干燥的 $10 \mathrm{~mL}$ 圆底烧瓶中加入离子液体 $1 \bullet\left(\mathrm{HSO}_{4}\right)_{2}(1.55 \mathrm{~g}, 3.5 \mathrm{mmol}), \mathrm{H}_{2} \mathrm{SO}_{4}(0.39 \mathrm{~g}, 4.0 \mathrm{mmol})$, $\mathrm{H}_{2} \mathrm{O}(18 \mathrm{mg}, 1.0 \mathrm{mmol})$ 以及 $0.5 \mathrm{mmol}$ 卤代苯乙炔, 置于 $70{ }^{\circ} \mathrm{C}$ 油浴锅中摚拌反应，薄层色谱(TLC) 监测反应进 度, $2 \mathrm{~h}$ 反应结束. 待冷却至室温后, 用 $5 \mathrm{~mL}$ 乙酸乙酯萃 取三次, 收集有机相旋转蒸发除去乙酸乙酯后经柱层析 [乙酸乙酯/石油醚]纯化分离得到产物.

离子液体催化体系循环方法

在干燥的 $100 \mathrm{~mL}$ 圆底烧瓶中加入离子液体 $\mathbf{1} \cdot\left(\mathrm{HSO}_{4}\right)_{2}(31.0 \mathrm{~g}, 70 \mathrm{mmol}), \mathbf{1 a}(1.81 \mathrm{~g}, 10 \mathrm{mmol}), \mathrm{H}_{2} \mathrm{O}$ (360 mg, $20 \mathrm{mmol}), \mathrm{H}_{2} \mathrm{SO}_{4}(7.84 \mathrm{~g}, 80 \mathrm{mmol})$, 置于 $70{ }^{\circ} \mathrm{C}$ 油浴锅中搅拌反应，薄层色谱(TLC)监测反应进度，反 应结束待冷却至室温后, 用 $50 \mathrm{~mL}$ 乙酸乙酯萃取产物三 次用于反应收率计算, 收集离子液体 $-\mathrm{H}_{2} \mathrm{SO}_{4}$ 体系并于 真空干燥箱中, $60{ }^{\circ} \mathrm{C} 、-0.09 \mathrm{MPa}$ (相对真空度), 真空干 燥 $24 \mathrm{~h}$, 充分干燥后直接用于下一次循环.

$\alpha$-溴代苯乙酮 $(\mathbf{2 a})^{[6]}$ : 展开剂: $V$ (乙酸乙酯) $: V($ 石油 醚) $=1: 40,90.5 \mathrm{mg}$, 收率 $91 \%$. 无色晶体, m.p. 50 $51{ }^{\circ} \mathrm{C}$ (lit. ${ }^{[10]} 50 \sim 51{ }^{\circ} \mathrm{C}$ ); ${ }^{1} \mathrm{H}$ NMR $\left(400 \mathrm{MHz}, \mathrm{CDCl}_{3}\right) \delta$ : $7.91(\mathrm{~d}, J=7.2 \mathrm{~Hz}, 2 \mathrm{H}), 7.56 \sim 7.52(\mathrm{~m}, 1 \mathrm{H}), 7.44 \sim 7.40$ $(\mathrm{m}, 2 \mathrm{H}), 4.38(\mathrm{~s}, 2 \mathrm{H}) ;{ }^{13} \mathrm{C}$ NMR $\left(100 \mathrm{MHz}, \mathrm{CDCl}_{3}\right) \delta$ : 191.3, 134.0, 133.9, 128.9, 128.8, 30.9.

$\alpha$-溴代-4-甲基苯乙酮 $(\mathbf{2 b})^{[11]}$ : 展开剂： $V($ 乙酸乙 酯) $: V$ (石油醚 $)=1: 40,99.0 \mathrm{mg}$, 收率 $93 \%$. 无色晶体, m.p. $51 \sim 52{ }^{\circ} \mathrm{C}$ (lit. ${ }^{[10]} 50 \sim 51{ }^{\circ} \mathrm{C}$ ); ${ }^{1} \mathrm{H}$ NMR $(400 \mathrm{MHz}$, $\left.\mathrm{CDCl}_{3}\right) \delta: 7.81(\mathrm{~d}, J=8.4 \mathrm{~Hz}, 2 \mathrm{H}), 7.22(\mathrm{~d}, J=8.0 \mathrm{~Hz}$, $2 \mathrm{H}), 4.35$ (s, 2H), $2.36(\mathrm{~s}, 3 \mathrm{H}) ;{ }^{13} \mathrm{C}$ NMR $(100 \mathrm{MHz}$, $\left.\mathrm{CDCl}_{3}\right) \delta: 190.9,145.0,131.3,129.5,129.0,31.0,21.7$.

$\alpha$-溴代-4-甲氧基苯乙酮 $(\mathbf{2 c})^{[12]}$ : 展开剂: $V$ (乙酸乙 酯) $: V$ (石油醚 $)=1: 40,101.9 \mathrm{mg}$, 收率 $89 \%$. 无色晶 体, m.p. $73 \sim 74{ }^{\circ} \mathrm{C}$ (lit. $\left.{ }^{[5]} 73 \sim 74{ }^{\circ} \mathrm{C}\right) ;{ }^{1} \mathrm{H}$ NMR $(400$ $\left.\mathrm{MHz}, \mathrm{CDCl}_{3}\right) \delta: 7.89(\mathrm{~d}, J=8.8 \mathrm{~Hz}, 2 \mathrm{H}), 6.88(\mathrm{~d}, J=8.8$ $\mathrm{Hz}, 2 \mathrm{H}), 4.32$ (s, 2H), 3.81 (s, 3H); ${ }^{13} \mathrm{C}$ NMR (100 MHz, $\left.\mathrm{CDCl}_{3}\right) \delta: 189.9,164.1,131.3,126.9,114.0,55.5,30.7$.

$\alpha$-溴代-4-苯基苯乙酮 $(\mathbf{2 d})^{[6]}$ : 展开剂： $V($ 乙酸乙 酯)：V(石油醚) $=1 ： 40,123.8 \mathrm{mg}$, 收率 $90 \%$. 无色晶 体, m.p. $126 \sim 127{ }^{\circ} \mathrm{C}$ (lit. ${ }^{[13]} 126.3 \sim 127{ }^{\circ} \mathrm{C}$ ); ${ }^{1} \mathrm{H}$ NMR $\left(400 \mathrm{MHz}, \mathrm{CDCl}_{3}\right) \delta: 7.99(\mathrm{~d}, J=7.6 \mathrm{~Hz}, 2 \mathrm{H}), 7.64(\mathrm{~d}, J=$ $7.6 \mathrm{~Hz}, 2 \mathrm{H}), 7.56(\mathrm{~d}, J=7.2 \mathrm{~Hz}, 2 \mathrm{H}), 7.42 \sim 7.39(\mathrm{~m}$, $2 \mathrm{H}), 7.36 \sim 7.32(\mathrm{~m}, 1 \mathrm{H}), 4.40(\mathrm{~s}, 2 \mathrm{H}) ;{ }^{13} \mathrm{C}$ NMR $(100$ 
$\left.\mathrm{MHz}, \mathrm{CDCl}_{3}\right) \delta: 190.9,146.6,139.5,132.6,129.5,129.0$, 128.5, 127.4, 127.3, 30.8.

$\alpha$-溴代-4-乙酰氧基苯乙酮 $(2 \mathrm{e})^{[14]}$ : 展开剂: $V($ 乙酸 乙酯)： $V$ (石油醚 $)=1: 20,111.8 \mathrm{mg}$, 收率 $87 \%$. 无色晶 体, m.p. $66 \sim 67{ }^{\circ} \mathrm{C}$ (lit. $\left.{ }^{[15]} 67{ }^{\circ} \mathrm{C}\right) ;{ }^{1} \mathrm{H}$ NMR $(400 \mathrm{MHz}$, $\left.\mathrm{CDCl}_{3}\right) \delta: 7.96(\mathrm{~d}, J=8.4 \mathrm{~Hz}, 2 \mathrm{H}), 7.16(\mathrm{~d}, J=8.4 \mathrm{~Hz}$, 2H), 4.36 (s, 2H), 2.26 (s, 3H); ${ }^{13} \mathrm{C}$ NMR (100 MHz, $\left.\mathrm{CDCl}_{3}\right) \delta: 190.1,168.7,154.9,131.4,130.6,122.1,30.6$, 21.1.

$\alpha$-澳代-4-氟苯乙酮 $(\mathbf{2} \mathbf{f})^{[11]}$ : 展开剂: $V$ (乙酸乙酯) : $V$ (石油醚) $=1: 20,95.5 \mathrm{mg}$, 收率 $88 \%$. 无色晶体, m.p. $49 \sim 50 \quad{ }^{\circ} \mathrm{C} \quad\left(\right.$ lit. $\left.{ }^{[5]} 49 \sim 50{ }^{\circ} \mathrm{C}\right) ;{ }^{1} \mathrm{H}$ NMR $(400 \mathrm{MHz}$, $\left.\mathrm{CDCl}_{3}\right) \delta: 7.99 \sim 7.90(\mathrm{~m}, 2 \mathrm{H}), 7.12 \sim 7.08(\mathrm{~m}, 2 \mathrm{H}), 4.34$ (s, 2H); ${ }^{13} \mathrm{C}$ NMR $\left(100 \mathrm{MHz}, \mathrm{CDCl}_{3}\right) \delta: 189.8,131.8$, 131.7, 130.9, 130.3, 116.2, 116.0, 30.4 .

$\alpha$-溴代-4-氯苯乙酮 $(\mathbf{2 g})^{[7 \mathrm{a}]}$ : 展开剂: $V$ (乙酸乙酯) : $V($ 石油醚 $)=1: 20,103.7 \mathrm{mg}$, 收率 $89 \%$. 无色晶体, m.p. 96 97 ${ }^{\circ} \mathrm{C} \quad\left(\right.$ lit. $\left.{ }^{[15]} 96 \sim 97{ }^{\circ} \mathrm{C}\right) ;{ }^{1} \mathrm{H}$ NMR $(400 \mathrm{MHz}$, $\left.\mathrm{CDCl}_{3}\right) \delta: 7.86(\mathrm{~d}, J=8.4 \mathrm{~Hz}, 2 \mathrm{H}), 7.40(\mathrm{~d}, J=8.4 \mathrm{~Hz}$, $2 \mathrm{H}), 4.33$ (s, $2 \mathrm{H}) ;{ }^{13} \mathrm{C}$ NMR (100 MHz, $\left.\mathrm{CDCl}_{3}\right) \delta: 190.2$, 140.5, 132.2, 130.4, 129.2, 30.3.

$\alpha$-澳代-4-碘苯乙酮 $(\mathbf{2 h})^{[16]}$ : 展开剂: $V$ (乙酸乙酯) : $V($ 石油醚 $)=1: 20,143.0 \mathrm{mg}$, 收率 $88 \%$. 无色晶体, m.p. $113 \sim 114{ }^{\circ} \mathrm{C}$ (lit. ${ }^{[17]} 113.5{ }^{\circ} \mathrm{C}$ ); ${ }^{1} \mathrm{H}$ NMR $(400 \mathrm{MHz}$, $\left.\mathrm{CDCl}_{3}\right) \delta: 7.80(\mathrm{~d}, J=8.0 \mathrm{~Hz}, 2 \mathrm{H}), 7.62(\mathrm{~d}, J=8.0 \mathrm{~Hz}$, 2H), $4.32(\mathrm{~s}, 2 \mathrm{H}) ;{ }^{13} \mathrm{C}$ NMR (100 MHz, $\left.\mathrm{CDCl}_{3}\right) \delta: 190.7$, 138.2, 133.1, 130.2, 102.2, 30.3.

$\alpha$-溴代-4-三氟甲基苯乙酮(2i) ${ }^{[7 \mathrm{a}]}$ : 展开剂: $V$ (乙酸乙 酯) : $V$ (石油醚) $=1: 20,114.8 \mathrm{mg}$, 收率 $86 \%$. 无色油状 物; ${ }^{1} \mathrm{H}$ NMR (400 MHz, $\left.\mathrm{CDCl}_{3}\right) \delta: 8.03(\mathrm{~d}, J=8.0 \mathrm{~Hz}$, $2 \mathrm{H}), 7.70$ (d, $J=8.0 \mathrm{~Hz}, 2 \mathrm{H}), 4.38$ (s, 2H); ${ }^{13} \mathrm{C}$ NMR $(101$ $\left.\mathrm{MHz}, \mathrm{CDCl}_{3}\right) \delta: 190.4,136.6,135.3,135.0,129.3,125.9$, 30.3 .

$\alpha$-溴代-4-硝基苯乙酮 $(\mathbf{2 j})^{[7 \mathrm{a}]}$ : 展开剂: $V($ 乙酸乙 酯)： $V$ (石油醚) $=1: 20,106.1 \mathrm{mg}$, 收率 $87 \%$. 无色晶 体, m.p. 95 97 ${ }^{\circ} \mathrm{C}$ (lit. ${ }^{[18]} 98{ }^{\circ} \mathrm{C}$ ); ${ }^{1} \mathrm{H}$ NMR $(400 \mathrm{MHz}$, $\left.\mathrm{CDCl}_{3}\right) \delta: 8.35(\mathrm{~d}, J=8.4 \mathrm{~Hz}, 2 \mathrm{H}), 8.17(\mathrm{~d}, J=8.4 \mathrm{~Hz}$, $2 \mathrm{H}), 4.47(\mathrm{~s}, 2 \mathrm{H}) ;{ }^{13} \mathrm{C}$ NMR (100 MHz, $\left.\mathrm{CDCl}_{3}\right) \delta: 189.9$, 150.7, 138.3, 130.1, 124.0, 30.1.

$\alpha$-溴代-4-氧基苯乙酮 $(\mathbf{2 k})^{[7 \mathrm{7}]}$ : 展开剂: $V($ 乙酸乙 酯) : $V$ (石油醚) $=1: 20,96.3 \mathrm{mg}$, 收率 $86 \%$. 淡黄色固 体, m.p. 92 93 ${ }^{\circ} \mathrm{C}$ (lit. $\left.{ }^{[19]} 92 \sim 94{ }^{\circ} \mathrm{C}\right) ;{ }^{1} \mathrm{H}$ NMR $(400$ $\left.\mathrm{MHz}, \mathrm{CDCl}_{3}\right) \delta: 8.02(\mathrm{~d}, J=7.6 \mathrm{~Hz}, 2 \mathrm{H}), 7.74(\mathrm{~d}, J=8.0$ $\mathrm{Hz}, 2 \mathrm{H}), 4.37$ (s, 2H); ${ }^{13} \mathrm{C}$ NMR (100 MHz, $\left.\mathrm{CDCl}_{3}\right) \delta$ : 190.1, 136.9, 132.7, 129.4, 117.6, 117.1, 30.0. $\alpha$-澳代-3-溴苯乙酮 $(21){ }^{[14]}$ : 展开剂: $V$ (乙酸乙酯) : $V($ 石油醚 $)=1: 20,122.3 \mathrm{mg}$, 收率 $88 \%$. 无色晶体, m.p. $51 \sim 52{ }^{\circ} \mathrm{C} \quad\left(\right.$ lit. $\left.{ }^{[5]} 51 \sim 52{ }^{\circ} \mathrm{C}\right) ;{ }^{1} \mathrm{H}$ NMR $(400 \mathrm{MHz}$, $\left.\mathrm{CDCl}_{3}\right) \delta: 8.03(\mathrm{~s}, 1 \mathrm{H}), 7.82(\mathrm{~d}, J=7.6 \mathrm{~Hz}, 1 \mathrm{H}), 7.66(\mathrm{~d}, J$ $=8.0 \mathrm{~Hz}, 1 \mathrm{H}), 7.32 \sim 7.28(\mathrm{~m}, 1 \mathrm{H}), 4.34(\mathrm{~s}, 2 \mathrm{H}) ;{ }^{13} \mathrm{C} \mathrm{NMR}$ $\left(100 \mathrm{MHz}, \mathrm{CDCl}_{3}\right) \delta: 190.0,136.7,135.6,131.8,130.4$, 127.4, 123.1, 30.5.

$\alpha$-溴代-3-甲基苯乙酮 $(\mathbf{2 m})^{[11]}$ : 展开剂: $V($ 乙酸乙 酯)：V(石油醚) $=1: 40,96.9 \mathrm{mg}$, 收率 $91 \%$. 无色晶体, m.p. $47 \sim 48{ }^{\circ} \mathrm{C}$ (lit. $\left.{ }^{[5]} 47 \sim 48{ }^{\circ} \mathrm{C}\right) ;{ }^{1} \mathrm{H}$ NMR $(400 \mathrm{MHz}$, $\left.\mathrm{CDCl}_{3}\right) \delta: 7.70(\mathrm{~d}, J=11.2 \mathrm{~Hz}, 2 \mathrm{H}), 7.36 \sim 7.28(\mathrm{~m}, 2 \mathrm{H})$, 4.37 (s, 2H), 2.35 (s, $3 \mathrm{H}) ;{ }^{13} \mathrm{C}$ NMR $\left(100 \mathrm{MHz}, \mathrm{CDCl}_{3}\right) \delta$ : 191.4, 138.8, 134.7, 134.0, 129.3, 128.7, 126.1, 31.0, 21.3.

$\alpha$-溴代-3-羟基苯乙酮 $(\mathbf{2 n})^{[76]}$ : 展开剂: $V($ 乙酸乙 酯)： $V$ (石油醚 $)=1: 20,95.7 \mathrm{mg}$, 收率 $89 \%$. 淡黄色油 状物; ${ }^{1} \mathrm{H}$ NMR $\left(400 \mathrm{MHz}, \mathrm{CDCl}_{3}\right) \delta: 7.43(\mathrm{~d}, J=7.6 \mathrm{~Hz}$, $2 \mathrm{H}), 7.30 \sim 7.26(\mathrm{~m}, 1 \mathrm{H}), 7.07(\mathrm{~d}, J=7.2 \mathrm{~Hz}, 1 \mathrm{H}), 6.79(\mathrm{~s}$, 1H), $4.39(\mathrm{~s}, 2 \mathrm{H}) ;{ }^{13} \mathrm{C}$ NMR $\left(100 \mathrm{MHz}, \mathrm{CDCl}_{3}\right) \delta: 192.1$, 156.5, 135.2, 130.2, 121.7, 121.3, 115.3, 31.2.

$\alpha$-溴代-2-甲基苯乙酮 $(\mathbf{2 0})^{[11]}$ : 展开剂: $V($ 乙酸乙 酯)：V(石油醚 $)=1: 40,95.9 \mathrm{mg}$, 收率 $90 \%$.淡黄色油 状物. ${ }^{1} \mathrm{H}$ NMR (400 MHz, $\left.\mathrm{CDCl}_{3}\right) \delta: 7.60(\mathrm{~d}, J=8.0 \mathrm{~Hz}$, $1 \mathrm{H}), 7.38 \sim 7.34(\mathrm{~m}, 1 \mathrm{H}), 7.23(\mathrm{~d}, J=7.2 \mathrm{~Hz}, 2 \mathrm{H}), 4.35(\mathrm{~s}$, $2 \mathrm{H}), 2.46(\mathrm{~s}, 3 \mathrm{H}) ;{ }^{13} \mathrm{C}$ NMR (100 MHz, $\left.\mathrm{CDCl}_{3}\right) \delta: 194.2$, 139.7, 134.4, 132.3, 132.3, 130.0, 125.8, 33.7, 21.4.

$\alpha$-溴代-2-氯苯乙酮 $(\mathbf{2} \mathbf{p})^{[6]}$ : 展开剂: $V($ 乙酸乙酯) : $V($ 石油醚 $)=1: 40,106.0 \mathrm{mg}$, 收率 $91 \%$. 淡黄色油状物; ${ }^{1} \mathrm{H}$ NMR $\left(400 \mathrm{MHz}, \mathrm{CDCl}_{3}\right) \delta: 7.50(\mathrm{~d}, J=7.6 \mathrm{~Hz}, 1 \mathrm{H})$, $7.38(\mathrm{~d}, J=4.0 \mathrm{~Hz}, 2 \mathrm{H}), 7.32 \sim 7.27(\mathrm{~m}, 1 \mathrm{H}), 4.45(\mathrm{~s}, 2 \mathrm{H})$; ${ }^{13} \mathrm{C} \mathrm{NMR}\left(100 \mathrm{MHz}, \mathrm{CDCl}_{3}\right) \delta: 194.0,136.2,132.7,131.3$, 130.6, 130.2, 127.1, 34.5.

2-澳-2-䒬乙酮 $(2 \mathbf{q})^{[7 \mathrm{7a}]}$ : 展开剂: $V$ (乙酸乙酯) $: V($ 石 油醚 $)=1: 40,112.0 \mathrm{mg}$, 收率 $90 \%$. 淡黄色油状物; ${ }^{1} \mathrm{H}$ NMR (400 MHz, $\left.\mathrm{CDCl}_{3}\right) \delta: 8.43(\mathrm{~s}, 1 \mathrm{H}), 7.96 \sim 7.89(\mathrm{~m}$, $2 \mathrm{H}), 7.87 \sim 7.78(\mathrm{~m}, 2 \mathrm{H}), 7.57 \sim 7.48(\mathrm{~m}, 2 \mathrm{H}), 4.51(\mathrm{~s}$, $2 \mathrm{H}) ;{ }^{13} \mathrm{C}$ NMR (100 MHz, $\left.\mathrm{CDCl}_{3}\right) \delta: 191.3,135.9,132.4$, $131.3,131.0,129.7,129.0,128.8,127.8,127.1,124.1$, 30.9 .

1-(1,3-苯并氧)2-溴乙酮 $(2 \mathbf{r})^{[20]}$ : 展开剂: $V($ 乙酸乙 酯)： $V$ (石油醚 $)=1: 40,106.9 \mathrm{mg}$, 收率 $88 \%$. 白色固 体, m.p. 86 87 ${ }^{\circ} \mathrm{C}$ (lit. ${ }^{[20]} 88{ }^{\circ} \mathrm{C}$ ); ${ }^{1} \mathrm{H}$ NMR $(400 \mathrm{MHz}$, $\left.\mathrm{CDCl}_{3}\right) \delta: 7.51(\mathrm{~d}, J=8.4 \mathrm{~Hz}, 1 \mathrm{H}), 7.38(\mathrm{~s}, 1 \mathrm{H}), 6.80(\mathrm{~d}$, $J=8.4 \mathrm{~Hz}, 1 \mathrm{H}), 5.99$ (s, 2H), 4.30 (s, 2H). ${ }^{13} \mathrm{C}$ NMR $(100$ $\left.\mathrm{MHz}, \mathrm{CDCl}_{3}\right) \delta: 189.5,152.5,148.4,128.6,125.6,108.6$, 108.1, 102.1, 30.5. 
$\alpha$-氯代苯乙酮 $(2 \mathrm{~s})^{[6]}$ : 展开剂: $V($ 乙酸乙酯) $: V$ (石油 醚) $=1: 40,72.4 \mathrm{mg}$, 收率 94\%. 无色晶体, m.p. 56 $58{ }^{\circ} \mathrm{C}$ (lit. $\left.{ }^{[5]} 57 \sim 58{ }^{\circ} \mathrm{C}\right) ;{ }^{1} \mathrm{H}$ NMR $\left(400 \mathrm{MHz}, \mathrm{CDCl}_{3}\right) \delta$ : $7.95(\mathrm{~d}, J=7.2 \mathrm{~Hz}, 2 \mathrm{H}), 7.63 \sim 7.59(\mathrm{~m}, 1 \mathrm{H}), 7.51 \sim 7.47$ $(\mathrm{m}, 2 \mathrm{H}), 4.72(\mathrm{~s}, 2 \mathrm{H}) ;{ }^{13} \mathrm{C}$ NMR $\left(100 \mathrm{MHz}, \mathrm{CDCl}_{3}\right) \delta$ : 191.1, 134.2, 134.0, 128.9, 128.5, 46.1.

$\alpha$-碘代苯乙酮 $(\mathbf{2 t})^{[7 b]}$ : 展开剂: $V$ (乙酸乙酯) $: V$ (石油 醚) $=1: 40,104.5 \mathrm{mg}$, 收率 85\%. 无色油状物; ${ }^{1} \mathrm{H}$ NMR $\left(400 \mathrm{MHz}, \mathrm{CDCl}_{3}\right) \delta: 7.91(\mathrm{~d}, J=7.6 \mathrm{~Hz}, 2 \mathrm{H}), 7.54 \sim 7.50$ (m, 1H), 7.43 7.39 (m, 2H), $4.29(\mathrm{~s}, 2 \mathrm{H}) ;{ }^{13} \mathrm{C}$ NMR $(100$ $\left.\mathrm{MHz}, \mathrm{CDCl}_{3}\right) \delta: 192.8,133.8,133.428,129.0,128.8$.

1-溴-6-乙酰氧基-2-己酮 $(\mathbf{2 u})^{[7 b]}$ : 展开剂: $V$ (乙酸乙 酯) : $V$ (石油醚 $)=1: 20,105.5 \mathrm{mg}$, 收率 $89 \%$. 无色油 状物; ${ }^{1} \mathrm{H}$ NMR $\left(400 \mathrm{MHz}, \mathrm{CDCl}_{3}\right) \delta: 4.00(\mathrm{t}, \quad J=6.0 \mathrm{~Hz}$, 2H), 3.83 (s, 2H), 2.64 (t, $J=6.8 \mathrm{~Hz}, 2 \mathrm{H}), 1.98$ (s, 3H), $1.63 \sim 1.59(\mathrm{~m}, 4 \mathrm{H}) ;{ }^{13} \mathrm{C}$ NMR $\left(100 \mathrm{MHz}, \mathrm{CDCl}_{3}\right) \delta$ : 201.6, 171.1, 63.8, 39.0, 34.1, 27.7, 20.9, 20.1.

2-澳-1-环己基乙酮 $(\mathbf{2 v})^{[7 \mathrm{~b}]}$ : 展开剂: $V$ (乙酸乙酯) : $V($ 石油醚 $)=1: 40,89.2 \mathrm{mg}$, 收率 $87 \%$. 无色油状物; ${ }^{1} \mathrm{H}$ NMR (400 MHz, $\left.\mathrm{CDCl}_{3}\right) \delta: 3.92$ (s, 2H), 2.64 (t, $J=11.2$ $\mathrm{Hz}, 1 \mathrm{H}), 1.81(\mathrm{~d}, J=13.2 \mathrm{~Hz}, 2 \mathrm{H}), 1.73(\mathrm{~d}, J=12.4 \mathrm{~Hz}$, $2 \mathrm{H}), 1.61(\mathrm{~d}, J=10.4 \mathrm{~Hz}, 1 \mathrm{H}), 1.36 \sim 1.16(\mathrm{~m}, 5 \mathrm{H})$.

3 -澳乙酰基噻吩 $(\mathbf{2 w})^{[6]}$ : 展开剂: $V$ (乙酸乙酯) : $V($ 石油醚 $)=1: 20,87.1 \mathrm{mg}$, 收率 $85 \%$. 无色晶体, m.p. $62 \sim 63{ }^{\circ} \mathrm{C} \quad\left(\right.$ lit. $\left.^{[5]} 62 \sim 63{ }^{\circ} \mathrm{C}\right) ;{ }^{1} \mathrm{H}$ NMR $(400 \mathrm{MHz}$, $\left.\mathrm{CDCl}_{3}\right) \delta: 8.11(\mathrm{~s}, 1 \mathrm{H}), 7.51(\mathrm{~d}, J=5.2 \mathrm{~Hz}, 1 \mathrm{H}), 7.31 \sim$ $7.29(\mathrm{~m}, 1 \mathrm{H}), 4.27(\mathrm{~s}, 2 \mathrm{H}) ;{ }^{13} \mathrm{C} \mathrm{NMR}\left(100 \mathrm{MHz}, \mathrm{CDCl}_{3}\right) \delta$ : 185.5, 138.7, 133.8, 127.3, 126.9, 31.6.

辅助材料(Supporting Information) 化合物原始核磁 共振谱图. 这些材料可以免费从本刊网站(http://siocjournal.cn/)上下载.

\section{References}

[1] (a) Erian, A. W.; Sherif, S. M.; Gaber, H. M. Molecules 2003, 8, 793.

(b) Zhang, H. ; Wei, Q.; Wei, S.; Qu, J.; Wang, B. Eur. J. Org. Chem. 2016, 3373.

[2] De Kimpe, N.; Verhé, R. The Chemistry of $\alpha$-Haloketones,
$\alpha$-Haloaldehydes and $\alpha$-Haloimines, Ed.: Patai, S., Wiley, Chichester, UK, 1988, pp. 1 119.

[3] (a) Ostrowski, T.; Golankiewicz, B.; De Clercq, E.; Andrei, G.; Snoeck, R. Eur. J. Med. Chem. 2009, 44,3313.

(b) Conde, S.; Pérez, D. I.; Martínez, A.; Perez, C.; Moreno, F. J. J. Med. Chem. 2003, 46, 4631.

[4] (a) Morton, H. E.; Leanna, M. R. Tetrahedron Lett. 1993, 34, 4481. (b) Patil, R. D.; Joshi, G.; Adimurthy, S.; Ranu, B. C. Tetrahedron Lett. 2009, 50, 2529.

(c) Tanemura, K.; Suzuki, T.; Nishida, Y.; Satsumabayashi, K.; Horaguchi, T. Chem. Commun. 2004, 470.

(d) Meshram, H. M.; Reddy, P. N.; Vishnu, P.; Sadashiv, K.; Yadav, J. S. Tetrahedron Lett. 2006, 47, 991.

(e) Pravst, I.; Zupan, M.; Stavber, S. Tetrahedron 2008, 64, 5191.

(f) Kosower, E. M.; Wu, G. S. J. Org. Chem. 1963, 28, 633.

(g) Kajigaeshi, S.; Kakinami, T.; Moriwaki, M.; Fujisaki, S.; Maeno, K.; Okamoto, T. Synthesis 1988, 545.

(h) Dieter, R. K.; Nice, L. E.; Velu, S. E. Tetrahedron Lett. 1996, 37, 2377.

[5] Xie, L.; Wu, Y.; Yi, W.; Zhu, L.; Xiang, J.; He, W. J. Org. Chem. 2013, 78, 9190.

[6] Zou, H.; He, W.; Dong, Q.; Wang, R.; Yi, N.; Jiang, J.; Peng, D.; He, W. Eur. J. Org.Chem. 2016, 116.

[7] (a) Chen, Z.; Ye, D.; Ye, M.; Zhou, Z.; Li, S.; Liu, L. Tetrahedron Lett. 2014, 55, 1373.

(b) Zeng, M.; Huang, R.; Li, W.; Liu, X.; He, F.; Zhang, Y.; Xiao, F. Tetrahedron Lett. 2016, 72, 3818.

[8] (a) Olivier-Bourbigou, H.; Magna, L.; Morvan, D. Appl. Catal. A: Gen. 2010, 373, 1 .

(b) Hu, Y.; Guo, Z.; Lue, B. M.; Xu, X. J. Agric. Food Chem. 2009, 57, 3845 .

(c) Tzani, A.; Douka, A.; Papadopoulos, A.; Pavlatou, E. A.; Voutsas, E.; Detsi, A. ACS Sustainable Chem. Eng. 2013, 1, 1180.

[9] Wong, W.; Ho, K.; Lee, L.; Lam, K.; Zhou, Z.; Chan, T.; Wong, K. ACS Catal. 2011, 1, 116.

[10] Wei, Q. L.; Zhang, S. S.; Gao, J.; Li, W. H.; Xu, L. Z.; Yu, Z. G. Bioorg. Med. Chem. 2006, 14, 7146.

[11] Reddy Bodireddy, M.; Gangi, R. N. C. Synth. Commun. 2013, 43, 2603.

[12] Guan, X.; AlMisbaa, Z.; Huang, K. Arabian J. Chem. 2015, 8, 892.

[13] Sugiura, A.; Kepner, R. E.; Webb, A. D. J. Org. Chem. 1962, 27, 773.

[14] Jiang, Q.; Sheng, W.; Guo, C. Green Chem. 2013, 15, 2175

[15] Rosenmund, K. W. Chem. Ber. 1957, 90, 1922.

[16] Lapointe, D.; Markiewicz, T.; Whipp, C. J.; Toderian, A.; Fagnou, K. J. Org. Chem. 2011, 76,749.

[17] Judefind, W. L. J. Am. Chem. Soc. 1920, 42, 1043.

[18] Arrieta, A. Synth. Commun. 1984, 14, 939.

[19] Suzuki, M. Yakugaku Zasshi 1952, 72, 305.

[20] Antunes, H.; Fardelone, L. C.; Rodrigues, J. A. R.; Moran, P. J. S. Tetrahedron: Asymmetry 2004, 15, 2615. 